\title{
Stabilization of Photonic Microwave Generation in Vertical-Cavity Surface- Emitting Lasers with Optical Injection and Feedback
}

Hong, Yanhua; Ji, Songkun; Xue, Chenpeng; Valle, Angel; Spencer, Paul; Li, Hongqiang

\section{Journal of Lightwave Technology}

DOI:

10.1109/JLT.2018.2815581

Published: 01/10/2018

Peer reviewed version

Cyswllt i'r cyhoeddiad / Link to publication

Dyfyniad o'r fersiwn a gyhoeddwyd / Citation for published version (APA):

Hong, Y., Ji, S., Xue, C., Valle, A., Spencer, P., \& Li, H. (2018). Stabilization of Photonic Microwave Generation in Vertical-Cavity Surface-Emitting Lasers with Optical Injection and Feedback. Journal of Lightwave Technology, 36(19), 4347-4353.

https://doi.org/10.1109/JLT.2018.2815581

\footnotetext{
Hawliau Cyffredinol / General rights

Copyright and moral rights for the publications made accessible in the public portal are retained by the authors and/or other copyright owners and it is a condition of accessing publications that users recognise and abide by the legal requirements associated with these rights.

- Users may download and print one copy of any publication from the public portal for the purpose of private study or research.

- You may not further distribute the material or use it for any profit-making activity or commercial gain

- You may freely distribute the URL identifying the publication in the public portal?
}

Take down policy

If you believe that this document breaches copyright please contact us providing details, and we will remove access to the work immediately and investigate your claim. 


\title{
Stabilization of Photonic Microwave Generation in Vertical-Cavity Surface-Emitting Lasers with Optical Injection and Feedback
}

\author{
Songkun Ji, Chenpeng Xue, Angel Valle, Paul S. Spencer, Hongqiang Li, and Yanhua Hong, Member, \\ IEEE
}

\begin{abstract}
The effect of optical feedback on stability and linewidth of photonic microwave generated in an optically injected single-mode vertical-cavity surface-emitting laser has been investigated in both cases of single feedback and double feedback. The stability of photonic microwave is quantified by measuring the microwave frequency range using the long sweep time of the radio frequency spectrum analyzer. The effect of the feedback phase on the side peaks in the single feedback has also been studied. The experimental results show that both single feedback and double feedback can reduce the linewidth of photonic microwave and improve its stabilization. A narrow linewidth photonic microwave spectrum with suppressed side peaks has been achieved in single feedback configuration at the optimal feedback phase condition. The simulated results using the spin-flip model confirm that side peaks suppression in single optical feedback configuration is sensitive to the feedback phase. For non-optimal feedback phase conditions, single optical feedback can generate many side peaks which are related to external cavity. These side peaks can be suppressed by introducing the second feedback.
\end{abstract}

Index Terms - Microwave photonics, phase noise, optical feedback, vertical cavity surface emitting lasers.

\section{INTRODUCTION}

$\mathrm{M}$ ICROWAVE photonics technology has attracted significantly research interest due to its wide variety of applications [1-6]. The dominant application of microwave

Manuscript received January 29, 2018. This work was supported in part by the Sêr Cymru National Research Network in Advanced Engineering and Materials, in part by funded by the Ministerio de Economía y Competitividad (MINECO/FEDER, UE), Spain, under project TEC2015-65212-C3-1-P and in part by the National Natural Science Foundation of China under grant 61711530652, 61675154 and 61177078).

S. Ji, P. S. Spencer and Y. Hong are with the School of Electronic Engineering, Bangor University, Bangor LL57 1UT, U.K. (e-mail: eep40a@bangor.ac.uk; p.spencer@bangor.ac.uk; y.hong@bangor.ac.uk ).

C. Xue is with the School of Electronic Engineering, Bangor University, Bangor LL57 1UT, U.K, on leave from the School of Communication and Information Engineering, University of Electronic Science and Technology of China, Chengdu 611731, China (e-mail: chenpeng_xue@ 163.com)

A. Valle is with the Instituto de Física de Cantabria (CSIC-Univ. de Cantabria), Avda. Los Castros s/n, E39005 Santander, Spain (e-mail: valle@ifca.unican.es).

H. Li is with Tianjin Key Laboratory of Optoelectronic Detection Technology and Systems, School of Electronics and Information Engineering, Tianjin Polytechnic University, Tianjin 300387, China (e-mail: lihongqiang@tjpu.edu.cn). photonics is radio over fiber, which allows the microwave signal to be transmitted in optical fibers with no electro-magnetic interference and low propagation losses [7]. Microwave photonics technologies include photonic generation, processing, control and distribution of microwave and millimeter-wave (mm-wave) signals [4]. The first step of utilizing microwave photonics technology is microwave photonic generation. Many techniques have been proposed and demonstrated in generating photonic microwave, which includes direct modulation, optical heterodyne technique, external modulation, mode-locked semiconductor lasers, optoelectronic oscillator (OEO) and period one (P1) oscillation $[4,8]$. Among these techniques, photonic microwave generation based on P1 oscillation dynamics has many advantages, such as a nearly single sideband (SSB) spectrum, which minimizes the power penalty, low cost due to all-optical components configuration and widely tunable oscillation frequency far from its original relaxation resonance frequency [9-11]. Semiconductor lasers subject to optical injection, exhibit many forms of nonlinear dynamics. One of such dynamical behaviors is usually referred to as a P1 oscillation and occurs when a stable locked laser experiences a Hopf bifurcation, which generates two dominant frequencies: one is generated from the optical injection while the other one is emitted near the cavity resonance frequency [12]. A microwave signal appears at the output of photodetector and is generated from the beating of the two dominant frequencies of the optical signals. The P1 oscillation has been investigated in conventional distributed feedback (DFB) lasers [9-11] and quantum dot (QD) lasers [13-14]. Vertical-cavity surface-emitting lasers (VCSELs) are also found to be an excellent candidate for photonic microwave generation due to their many advantages, such as circular beam profile, single-longitudinal mode operation, ease of fabrication, low power consumption and low-cost [15]. In recent years, photonic microwave generation using VCSELs has drawn a great deal of attention [16-20]. More than $20 \mathrm{GHz}$ photonic microwave signal has been obtained using dual-beam orthogonal optical injection in a single-transverse-mode VCSEL [17]. Lin et al. also achieved photonic microwave in a multimode VCSEL subject to orthogonal optical injection [20]. In our previous work [19], we experimentally demonstrated photonic microwave generation utilizing single-mode 
VCSEL's nonlinear dynamical P1 oscillation with a tunable microwave frequency of over $15 \mathrm{GHz}$ (limited by the photo detector).

Despite the great achievements of photonic microwave generation based on P1 oscillations, P1 dynamics inherently contains phase noise due to the fluctuations in the lasers [21]. This phase noise is found to reduce the signal-to-noise ratio in communication [10]. Phase noise can also increase the microwave linewidth and the typical $3 \mathrm{~dB}$ linewidth of the generated microwave based on P1 dynamics is in the order of megahertz [12, 21-22], which affect the performance in RoF application [21] and the maximum detection range in Doppler velocimeters [23]. To address this issue, several techniques have been introduced to minimize the phase noise and reduce its linewidth, such as using double-locking with a microwave source [24-25], dual-beam optical injection [26-27], optoelectronic feedback [28], combining optical injection and polarization-rotated optical feedback [11] and optical feedback $[18,21,22,29]$. Optical feedback is a simple and low-cost method to achieve linewidth reduction. It has been theoretically and experimentally proved in DFB lasers [21-22, 29]. Dual feedback loops have also been used to reduce the linewidth of the generated photonic microwave in two- mode VCSEL [18]. However, to the best of our knowledge, no study has been reported the effect of the optical feedback on the stabilization of the broadly tunable photonic microwave in single-transverse mode VCSELs or the linewidth reduction via the control of the optical feedback phase in long external cavity.

In this paper, we study the effect of both single optical feedback and double optical feedback on the linewidth of the generated photonic microwave based P1 oscillation in a single-mode VCSEL. We also study the effect of the feedback phase on the side-peaks suppression. The results show that both single and double feedbacks can be used to reduce the linewidth and stabilize the microwave's fundamental frequency. The side peaks suppression in single feedback configuration was found to be feedback phase dependent. The paper is organized as follows. The experimental setup is described in Section 2, followed by the experimental results in section 3 . In section 4 , the numerical simulation of the feedback phase effect on the side peaks suppression is presented. Finally, we summarize the results in section 5 .

\section{EXPERIMENTAL SETUP}

The schematic of the experimental setup is shown in Fig. 1. The experiment was setup in free-space to overcome the uncertainty of injection and feedback polarization direction. A commercial single mode VCSEL (RayCan RC33xxx1-T) was used as the slave laser (SL). The VCSEL was driven by a low noise current source (Yokogawa GS200 DC Voltage/Current source) and its temperature was controlled to $21^{\circ} \mathrm{C}$. At this temperature, the threshold current of the VCSEL was $2.1 \mathrm{~mA}$. Near the threshold current, the VCSEL operated in one linear polarization (Y-polarization). When the bias current was increased to $2.4 \mathrm{~mA}$, the polarization direction switched to its orthogonal polarization direction (X-polarization). Further increases in the bias current to $5.7 \mathrm{~mA}$ resulted in the polarization direction switching back to the Y-polarization. The polarization remains in the Y-polarization state within the remaining operating current range. The VCSEL was operated at $8 \mathrm{~mA}$ (Y-polarization) through the experiment, unless stated otherwise. At this bias current, the VCSEL's lasing wavelength was $1559.15 \mathrm{~nm}$ and its output power was $1.87 \mathrm{~mW}$.

A tunable laser (Agilent 81682A installed on Agilent $8164 \mathrm{~A}$ ) was used as the master laser (ML). The maximum output power of the tunable laser is $10 \mathrm{~mW}$ and its linewidth is $1 \mathrm{MHz}$. The output of the ML passes-through an optical isolator to prevent the optical injection from the VCSEL and the optical feedback from the optical components. The polarization of the optical injection beam from the ML was adjusted by a half-wave plate $(\lambda / 2)$ to ensure the polarization of the injection beam is parallel to the free running VCSEL's polarization direction (Y-polarization). Excitation of X-polarization was not observed experimentally. The injection power was controlled by the ML's output power. The optical injection power $P_{\text {inj }}$ was measured just before the injection beam entered the VCSEL. It is noted that the actual injection power that the VCSEL received was less than the measurement due to the coupling loss. The tuning of the frequency detuning $\Delta f=f_{\mathrm{ML}}-f_{\mathrm{SL}}$ was achieved by changing the frequency of the ML. $f_{\mathrm{ML}}$ and $f_{\mathrm{SL}}$ are the frequencies of the free-running ML and the free-running VCSEL, respectively. Optical feedback cavities were created by mirror\#2 (Mir.\#2) and mirror\#3 (Mir.\#3), which are referred to as cavity 1 and 2, respectively. To study the linewidth of the generated photonic microwave with respect to the feedback phase, mirror\#3 was mounted on a piezo stage (PZT, PI-P752.1C1) to finely tune the cavity length. The feedback round trip times in the cavities formed by mirror\#2 and 3 are $7.3 \mathrm{~ns}\left(\tau_{L}\right)$ and $5.1 \mathrm{~ns}\left(\tau_{\mathrm{s}}\right)$, respectively. The feedback powers from both feedback cavities were the same and were adjusted by an optical attenuator (Atten.) and monitored by a power meter (PM). The output of the VCSEL was detected by a 12 $\mathrm{GHz}$ bandwidth photodetector (New Focus 1554-B) and recorded by a $30 \mathrm{GHz}$ RF spectrum analyzer (Anritsu MS2667C). The frequency resolution of the RF spectrum analyzer's was set at $10 \mathrm{kHz}$ and the sweep time was 50 milliseconds unless stated otherwise.

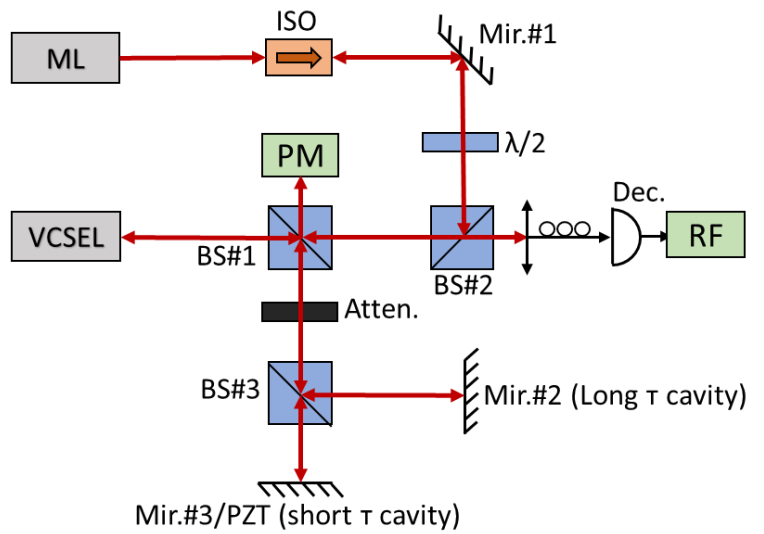

Fig. 1. The experimental setup. ML: Master laser, ISO: Optical isolator, Mir.: Mirror, $\lambda / 2$ : Half-wave plate, BS: Beam splitter, Atten: Optical attenuator, PM: Power meter, Dec: Photodetector, RF: Radio frequency spectrum analyzer, PZT: Piezo stage. 


\section{EXPERIMENTAL RESULTS}

The focus of this paper is the effect of optical feedback on the linewidth and stabilization of photonic microwave generated from the VCSEL's P1 dynamics. The P1 dynamics have previously been shown to exist over large injection parameters [19]. Two cases of optical feedback are studied. The first case is single feedback, which means that only optical feedback from one feedback cavity is allowed and the feedback from the other feedback cavity is blocked. The second case is double feedback, the feedback from both cavities 1 and 2 are re-entered the VCSEL.

\section{A. Single feedback}

\section{1) Single feedback and linewidth of microwave}

When the injection parameters were set at $\left(\Delta f, \mathrm{P}_{\text {inj }}\right)=(12.8$ $\mathrm{GHz}, 0.703 \mathrm{~mW}$ ), the VCSEL exhibited P1 dynamics and the fundamental frequency $f_{0}$ was $13.34 \mathrm{GHz}$, as shown in Fig. 2(a). The $3 \mathrm{~dB}$ linewidth $\left(\Delta \mathrm{f}_{0}{ }^{\prime}\right)$ of the generated photonic microwave was measured and found to be $4.62 \mathrm{MHz}$. When the optical feedback from one cavity was introduced into the VCSEL (cavity 2 was block) and the feedback power adjusted to $8 \mu \mathrm{W}$, the power spectrum shown in Fig. 2(b) was obtained. The linewidth has been found to have reduced to $1.13 \mathrm{MHz}$, however, many residual side peaks are observed and the spacing between side peaks is $141 \mathrm{MHz}$, which is very close to the reciprocal of the feedback delay time (7.3ns) of $137 \mathrm{MHz}$. If cavity 1 was now blocked and instead $8 \mu \mathrm{W}$ of feedback power introduced from cavity 2, the power spectrum of the VCSEL was modified as shown in Fig. 2(c). The spectrum is similar to Fig. 2(b), with multiple side peaks uniformly spaced at 198 $\mathrm{MHz}$, which is near to $1 / \tau_{\mathrm{s}}=196 \mathrm{MHz}$. Different injection conditions were also examined, and similar results were observed. This phenomenon is the same as that in the simulation results [21] and the experimental results of [22] for DFB lasers.

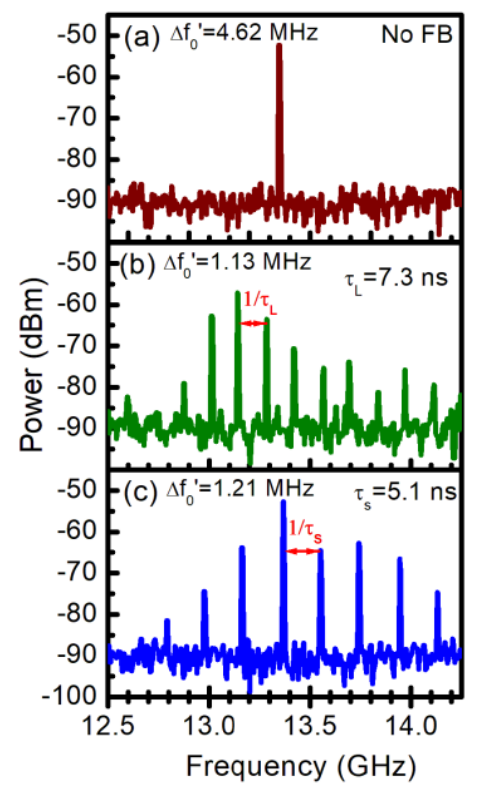

Fig. 2 Power spectra of the VCSEL. (a) without optical feedback, (b) with optical feedback from cavity 1 , (c) with optical feedback from cavity 2

\section{2) Feedback phase and side peaks suppression}

In this study, we also investigate the dynamics of the VCSEL with respect to the feedback phase. The feedback phase from cavity 2 is controlled by tuning the PZT stage distance. We fix the injection parameters $\left(\Delta f, \mathrm{P}_{\mathrm{inj}}\right)=(10.7 \mathrm{GHz}, 0.689 \mathrm{~mW})$ and feedback power $\mathrm{P}_{\mathrm{fb}}=8 \mu \mathrm{W}$. Fig. 3(a) is one of the representative power spectrum of the VCSEL when PZT stage distance is set between $1380 \mathrm{~nm}$ and $1890 \mathrm{~nm}$. The obtained signal exhibits multiple side peaks, the same phenomenon as described in Fig. 2(b) and (c). We refer to this condition as side peaks (SP) phase condition. When we move the PZT distance to $1890 \mathrm{~nm}$, the side peaks are suddenly suppressed and a very stable microwave signal is obtained, as shown in Fig. 3(b). This other condition we will refer to as side peaks suppression (SPS) phase condition. Further increases in the PZT distance have no effect on the power spectrum until the PZT distance reaches $2120 \mathrm{~nm}$. When the PTZ distance moves to $2120 \mathrm{~nm}$, the power spectrum of the VCSEL changes abruptly from SPS phase condition to SP phase condition. The SP phase condition persists for PTZ distance between $2120 \mathrm{~nm}$ and $2740 \mathrm{~nm}$, as shown in Fig. 3(c). The summarized different phase condition is presented in Fig. 3(d). The wine color and blue color represent the SPS phase condition and SP phase condition, respectively. From analyzing Fig. 3(d), it is found that side peaks are periodically suppressed when the external cavity length is increased. The period for the side peaks suppression is approximate to $750 \mathrm{~nm}$, which is about half of the lasing wavelength of the VCSEL, which corresponds to a $2 \pi$ feedback phase change.

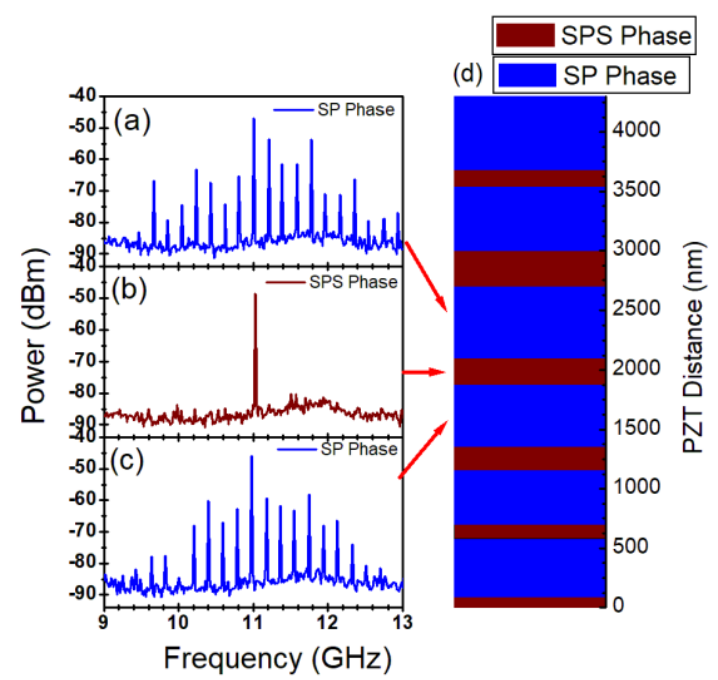

Fig. 3(a-c) Power spectra of the VCSEL with different PZT distance, (d) SPS phase condition and SP phase condition as a function of the PZT moving distance.

\section{B. Double feedback}

In this section, we study whether the side peaks under the single feedback with SP phase condition can be suppressed by adding the second feedback, just as those for DFB lasers [21-22]. Figure 4 shows that the power spectra of the optically injected VCSEL subject to optical feedback. The injection 
parameters $\left(\Delta f, \mathrm{P}_{\text {inj }}\right)$ are $(11.42 \mathrm{GHz}, 0.685 \mathrm{~mW})$. When the VCSEL is subject to single feedback from cavity 2 with the feedback power of $8 \mu \mathrm{W}$ and the PZT distance is tuned to SP phase condition, there are many side peaks around the fundamental frequency, as shown in Fig. 4(a). When optical feedback from cavity 1 is introduced and feedback power from each cavity set at $4 \mu \mathrm{W}$, the side peaks are suppressed in Fig. 4(b). The results indicate that the second feedback suppresses the side peaks. The side peaks suppression using double feedback is not unique to these specific injection parameters; the same phenomenon has also been observed across the period one dynamic region.

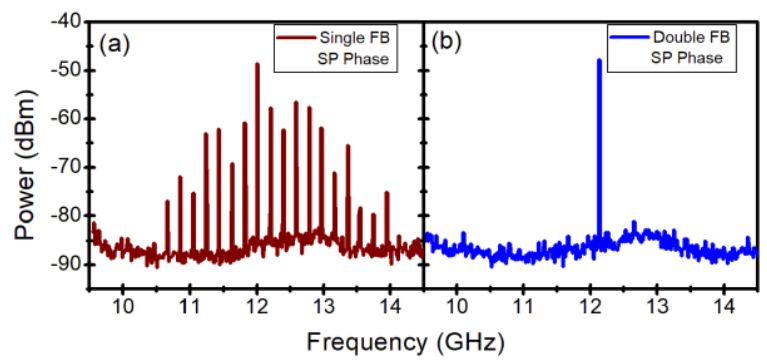

Fig. 4 Power spectra of the VCSEL with (a) single feedback, (b) double feedback.

\section{1) Feedback strength and linewidth of microwave}

The linewidth reduction through dual-cavity feedback with the different feedback power has also been investigated. Fig.5 shows the power spectrum of the VCSEL under the injection parameters of $\left(\Delta f, \mathrm{P}_{\mathrm{inj}}\right)=(12.8 \mathrm{GHz}, 0.772 \mathrm{~mW})$ with different feedback conditions. Figure 5(a) is for the power spectrum of the VCSEL with optical injection, but without optical feedback. The result indicates that the VCSEL operates in P1 dynamics with the fundamental frequency $f_{0}=13.5 \mathrm{GHz}$. The microwave $3 \mathrm{~dB}$ linewidth $\left(\Delta f_{0}{ }^{\prime}\right)$ was found to be $3.6 \mathrm{MHz}$. The linewidth reduction of the microwave signal has been seen when the combined optical feedback power from two cavities was set at 3 $\mu \mathrm{W}$, as shown in Fig. 5(b). The linewidth was reduced to 2.40 $\mathrm{MHz}$ and the fundamental frequency also increases slightly to $f_{0}$ $=13.7 \mathrm{GHz}$. When the total feedback power increases to $6 \mu \mathrm{W}$, a further linewidth reduction of $\Delta f_{0}{ }^{\prime}=1.62 \mathrm{MHz}$ can be achieved. Further increases in the feedback power to $9 \mu \mathrm{W}$, narrow the linewidth to $0.49 \mathrm{MHz}$, which is about one tenth of the linewidth without optical feedback. Increasing the feedback power beyond $9 \mu \mathrm{W}$ causes instability in the microwave generation, with multiple randomly spaced power peaks appearing in RF spectrum, which evidentially lead to the appearance of chaos dynamics.

The relationship between the microwave $3 \mathrm{~dB}$ linewidth and feedback strength is summarized in Fig. 6(a). The graph shows an almost inversed linear relationship between the linewidth and the feedback power. Fig. 6(b) shows the fundamental microwave frequency as a function of the feedback power. There is frequency shift of about $300 \mathrm{MHz}$ in the fundamental frequencies after applying the feedback. This is due to the decrease of the carrier density, which increased the refractive index, thus reducing the cavity resonant frequency [30]. As described in the introduction, the microwave signal is obtained by the beating between the regenerated injection beam and the red shift cavity frequency, therefore the microwave frequency increases with the optical feedback for the positive frequency detuning. Such frequency shift can be compensated for by adjusting the injection parameters.

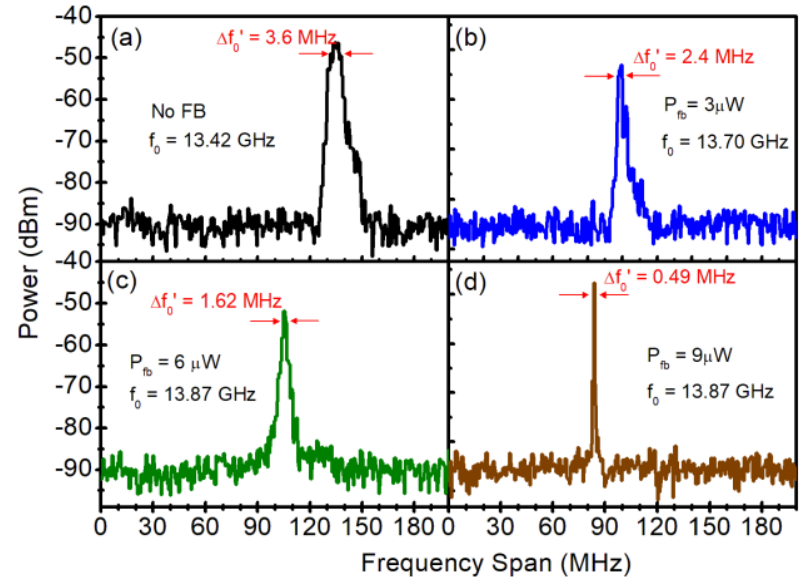

Fig. 5 Power spectra of the VCSEL. (a) Without optical feedback, (b-d) with double feedback and the total feedback powers are (b) $3 \mu \mathrm{W}$, (c) $6 \mu \mathrm{W}$, (d) 9 $\mu \mathrm{W}$.
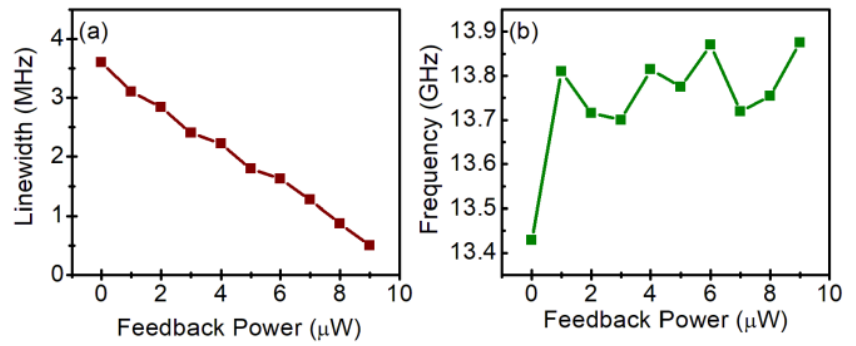

Fig. 6 (a) Linewidth and (b) fundamental frequency of the generated microwave as a function of the feedback power.

\section{2) Feedback strength and stability of microwave}

Readers may have noticed that the microwave fundamental frequencies do not always appear at the center of the frequency span in Fig.5, which is caused by fluctuation in the fundamental frequency. Practically, the fundamental frequency can randomly appear in the frequency range even at fixed injection parameters during the experiment. This behavior is highly correlated with the noise of the bias current, performance of the temperature controller and the accuracy of the injection tunable laser or the slight change of the injection coupling efficiency caused by the optical table vibrations during the experiment. However, in this experiment we have noticed that adding dual-cavity optical feedback also helps to improve the stability of the microwave fundamental frequency. To demonstrate this effect, the sweep time of the RF Spectrum analyzer is set to be 30 seconds, so the RF analyzer will capture multiple peaks during this timeframe and display them on a single image. The results can be found in Fig.7. With no feedback enabled, the location of the $f_{0}$ appears within $35.3 \mathrm{MHz}$ range. When the VCSEL is subject to optical feedback from both cavities with the total feedback power of $3 \mu \mathrm{W}$, the range of $f_{0}$ reduces to 24.7 MHz. Increasing the feedback power to $6 \mu \mathrm{W}$, and $f_{0}$ is 
captured within a $19.9 \mathrm{MHz}$ range. Further increases in the feedback power to the maximum allowed feedback power of 9 $\mu \mathrm{W}$, before unstable signal occurs, and the $f_{0}$ range is reduced to $9.4 \mathrm{MHz}$. The results show a significant reduction in the range of $f_{0}$ appearing with double feedback compared to that without optical feedback, which indicates that double feedback can increase the stability of the photonic microwave generated based on P1 dynamics.

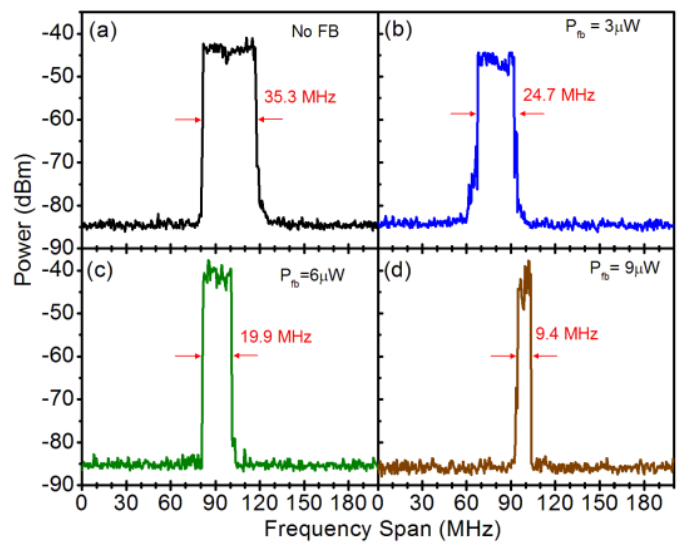

Fig. 7 Power spectra of the VCSEL when the sweep time of the RF spectrum analyzer is set at 30 seconds. (a) Without feedback, (b-d) with double feedback and the total feedback powers are (b) $3 \mu \mathrm{W}$, (c) $6 \mu \mathrm{W}$, (d) $9 \mu \mathrm{W}$

\section{3) Feedback phase and stability of microwave}

As described above, the side peaks suppression in the single feedback case is sensitive to the optical feedback phase. Adding a second feedback can suppress the side peaks for the SPS phase condition. In this section, we will examine whether the feedback with SPS phase condition in one feedback cavity has the same beneficial effect of linewidth reduction and stability as under double feedback. Fig. 8 illustrates the power spectra of VCSEL when the injection parameters and feedback power are $\left(\Delta f, \mathrm{P}_{\text {inj }}\right)=(11.42 \mathrm{GHz}, 0.685 \mathrm{~mW})$ and $8 \mu \mathrm{W}$, respectively. The left and right columns are for a sweep time of 50 milliseconds and 30 seconds in the RF spectrum analyzer, respectively. Fig. 8(a1) and (a2) are obtained when the VCSEL is subject to single feedback with the SPS phase condition. Fig. 8(a1) shows a linewidth of $0.453 \mathrm{MHz}$. When the sweep time of the RF spectrum analyzer is increased to 30 seconds, the power spectrum of the VCSEL is recorded and plotted in Fig. 8(a2). The result indicates that the fluctuation range of the fundamental frequency $f_{0}$ is about $14.2 \mathrm{MHz}$. When the second feedback is introduced we keep the total feedback power to 8 $\mu \mathrm{W}$. We can see that the linewidth has reduced to $0.296 \mathrm{MHz}$ for $50 \mathrm{~ms}$ sweep time, as shown in Fig. 8(b1), and the fluctuation range of the fundamental frequency $f_{0}$ has also decrease to $9.2 \mathrm{MHz}$, as indicated in Fig. 8(b2). When the PZT distance is adjusted to the SP phase condition from cavity 2 , the power spectra of the VCSEL from the short sweep time in Fig. 8(c1) and long sweep time in Fig. 8(c2), indicates that the fluctuation range of the fundamental frequency is very close to the double feedback cavity situation seen with the SPS phase condition, but has increased slightly to $9.6 \mathrm{MHz}$. However, the linewidth measured using the $50 \mathrm{~ms}$ sweep time has increased dramatically to $0.499 \mathrm{MHz}$. With a further enhanced experimental set-up it may be possible to further reduce the microwave linewidth by using a second PZT, but such the setup would increase the cost and reduce simplicity and is beyond the capability and scope of this current work. The results in Fig. 8 demonstrate that the second feedback can be used to reduce the linewidth and improve the stability of the generated microwave. Careful adjustment of the feedback phase can further decrease the linewidth.

Although the minimum suppressed linewidth by optical feedback is not yet as good as that using external modulation [28], the main advantage of the optical feedback scheme is that it is simple and low-cost and eliminates the need for high cost high frequency electronic devices to achieve the required linewidth reduction.

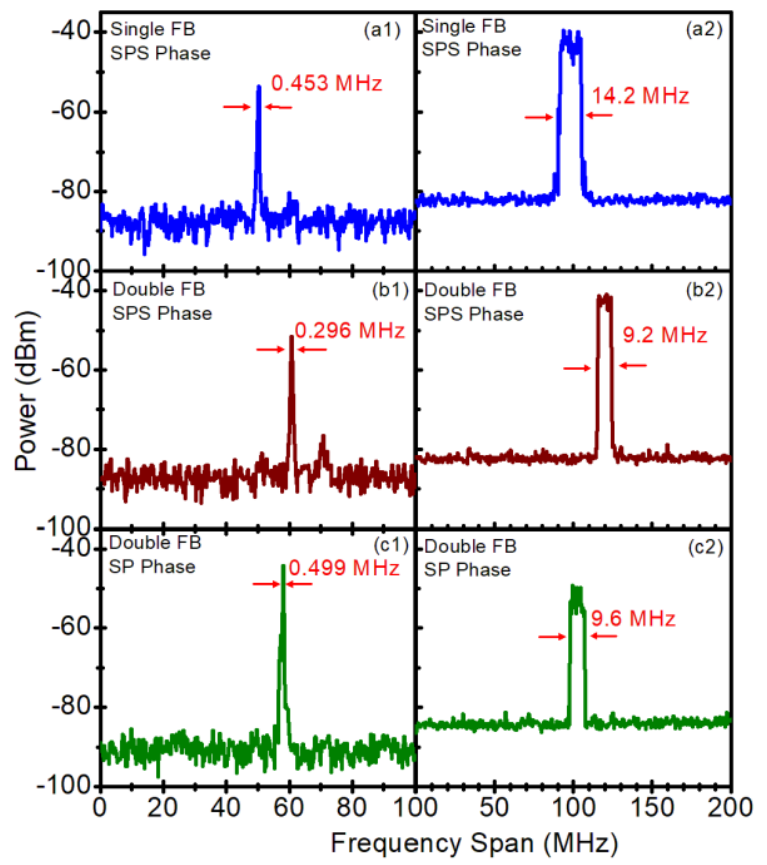

Fig. 8 The power spectra of the VCSEL with the different feedback configurations. The left and right columns are for the sweep time of the RF spectrum analyzer of 50 milliseconds and 30 seconds, respectively. (a1), (a2) single feedback with SPS phase condition, (b1), (b2) double feedback with SPS phase condition, (c1), (c2) double feedback with SP phase condition.

\section{NUMERICAL SIMULATION}

In order to check whether in-phase optical feedback or out-of-phase optical feedback favors the side peaks suppression in the single feedback configuration, the photonic microwave generated via P1 dynamics from optical feedback has been numerically simulated using the spin-flip model [18], as shown in Eqs (1)-(4) below.

$$
\begin{aligned}
\frac{d E_{x}}{d t}= & k(1+i \alpha)\left(N E_{x}-E_{x}+i n E_{y}\right) \\
& -\left(\gamma_{a}+i \gamma_{p}\right) E_{x}+\eta_{x} E_{i n j} e^{i 2 \pi\left(v_{i n j}-v\right) t} \\
& +\xi_{1} E_{x}\left(t-\tau_{1}\right) e^{-i\left(2 \pi v \tau_{1}+\phi\right)} \\
& +\xi_{2} E_{x}\left(t-\tau_{2}\right) e^{-i 2 \pi v \tau_{2}}+F_{x}
\end{aligned}
$$




$$
\begin{aligned}
\frac{d E_{\mathrm{y}}}{d t}= & k(1+i \alpha)\left(N E_{y}-E_{y}-i n E_{x}\right) \\
& +\left(\gamma_{a}+i \gamma_{p}\right) E_{y}+\eta_{y} E_{i n j} e^{i 2 \pi\left(v_{i n j}-v\right) t} \\
& +\xi_{1} E_{y}\left(t-\tau_{1}\right) e^{-i\left(2 \pi v \tau_{1}+\phi\right)} \\
& +\xi_{2} E_{y}\left(t-\tau_{2}\right) e^{-i 2 \pi v \tau_{2}}+F_{y} \\
\frac{d N}{d t}= & -\gamma_{e} N\left(1+\left|E_{x}\right|^{2}+\left|E_{x}\right|^{2}\right)+\gamma_{e} \mu \\
& -i \gamma_{e} n\left(E_{y} E_{x}^{*}-E_{x} E_{y}^{*}\right) \\
\frac{d n}{d t}= & -\gamma_{s} n-\gamma_{e} n\left(\left|E_{x}\right|^{2}+\left|E_{x}\right|^{2}\right) \\
& -i \gamma_{e} N\left(E_{y} E_{x}^{*}-E_{x} E_{y}^{*}\right)
\end{aligned}
$$

In the rate equations, the subscripts $x$ and $y$ stand for the $\mathrm{X}$ and Y polarizations of the VCSEL, respectively. $\mathrm{E}$ is the complex electric field; $\mathrm{N}$ is the total carrier density; $\alpha$ is the linewidth enhancement factor; $\gamma_{a}$ is the linear dichroism; $\gamma_{p}$ is the linear birefringence; $\gamma_{e}$ is the decay rate of the total carrier population; $n$ is the difference between the carrier densities with opposite spin values; $k$ is the field decay rate; $\gamma_{s}$ is the spin-flip rate; $\eta_{x}$ and $\eta_{y}$ are the injection strength into the $\mathrm{X}, \mathrm{Y}$ polarization direction; $E_{\text {inj }}$ is the injection field amplitude. $v$ is the central frequency of the free running VCSEL, $v=\left(v_{x}+v_{y}\right) / 2$, where $v_{x}$ and $v_{y}$ are the frequency of the VCSEL's X, Y polarization components. $v_{i n j}$ is the injection frequency; $\xi_{1}$ and $\xi_{2}$ are the feedback strength of each feedback cavity; $\tau_{1}$ and $\tau_{2}$ are the feedback delay time in each external cavity; $\mu$ is the normalized bias current. $F_{x}$ and $F_{y}$ are the Langevin noise, which are described below

$$
\begin{gathered}
F_{x}=\sqrt{\frac{\beta_{s p}}{2}}\left(\sqrt{N+n \zeta_{1}}+\sqrt{N-n \zeta_{2}}\right) \\
F_{y}=-i \sqrt{\frac{\beta_{s p}}{2}}\left(\sqrt{N+n \zeta_{1}}-\sqrt{N-n \zeta_{2}}\right)
\end{gathered}
$$

$\zeta_{1}$ and $\zeta_{2}$ are the independent Gaussian white noise sources with zero mean and unit variance and $\beta_{s p}$ is the noise strength. In the first feedback term in equations (1) and (2), we also add a phase $\phi$ in conjunction with phase change due to time delay $\tau_{1}$ to study the feedback phase effect. $\phi$ takes 10 equally spaced points within the range of $\phi \in[0,2 \pi]$.

A fourth order Runge-Kutta integration method is used to numerically solve Eqs. (1)-(4), where a temporal resolution of $\Delta t=1 \mathrm{ps}$ is selected and the duration of the time series is $1 \underline{\mu \mathrm{s}}$. The parameters used in the simulation are: $\alpha=3, \kappa=125 \mathrm{~ns}^{-1}, \gamma_{p}$ $=94.2 \mathrm{~ns}^{-1}, \gamma_{a}=0.5 \mathrm{~ns}^{-1}, \gamma_{e}=0.6 \mathrm{~ns}^{-1}, \gamma_{s}=140 \mathrm{~ns}^{-1}, \beta_{s p}=5 \times 10^{-5}$ $\mathrm{ns}^{-1}, \tau_{1}=5.1 \mathrm{~ns}, \tau_{2}=7.3 \mathrm{~ns}, v=193.55 \mathrm{THz}$ (corresponds to $1550 \mathrm{~nm}$ wavelength), $\mu=1.9$. With these parameters, the VCSEL lases in the Y-polarization state. For parallel injection, $\eta_{x}=0$. When $\eta_{\mathrm{y}}=50 \mathrm{GHz}$, the frequency detuning between the injection frequency and Y-polarization frequency is $10 \mathrm{GHz}$ $\left(v_{i n j}-v=v_{i n j}-v_{y}+\gamma_{p} / 2 \pi=25 \mathrm{GHz}\right)$, the VCSEL operates at P1 dynamics with the fundamental frequency of $11.21 \mathrm{GHz}$. Firstly, only single feedback is considered, $\xi_{1}=1.2 \mathrm{GHz}$ and $\xi_{2}$ $=0$. The power spectra of the VCSEL with the different phase are plotted with the black curves shown in Fig. 9. Figure 9(a) is for in-phase optical feedback $(\phi=0)$. The result shows that the fundamental frequency has shifted slightly to $11.31 \mathrm{GHz}$ with many evenly space side peaks. The spacing between the side peaks is about $190 \mathrm{MHz}$, which is slightly lower than $196 \mathrm{MHz}$ $\left(1 / \tau_{1}\right)$. The side peak suppression is $30.4 \mathrm{~dB}$. When the $\phi$ changes to $0.2 \pi$, more side peaks are excited [See Fig. 9(b)]. The side peak suppression has reduced to about $10.9 \mathrm{~dB}$. A further increasing $\phi$ to $0.4 \pi$, sees many side peaks still being observed with some improvement of side peak suppression to $21.2 \mathrm{~dB}$. When the VCSEL is subject to anti-phase feedback $(\phi=\pi)$, very few side peaks still exist, but the side peaks suppression is much higher and reaches $42.4 \mathrm{~dB}$. For the other feedback phase, the side peak suppression is between $10.9 \mathrm{~dB}$ and $42.4 \mathrm{~dB}$. So, for these parameters, the best side peak suppression is anti-phase feedback, the worst side peak suppression is the feedback phase of $0.2 \pi$. When we add the second feedback and set $\xi_{1}=\xi_{2}=0.6 \mathrm{GHz}$ to keep the total feedback strength the same as the single feedback, and keep the other parameters unchanged, we find that all side peaks have been suppressed, as displayed with the red curves in Fig. 9. The numerical results have shown good agreement with the experimental results obtained. It is noted that the side peaks are gradually suppressed with the changing feedback phase, which is different from the experimental results. It is also noted that the SPS phase condition is dependent on the injection parameters and the SPS phase condition happens at the different feedback phase for the different microwave frequency. The detailed study of the feedback phase on the side peaks suppression is complex and is beyond the scope of the current work.
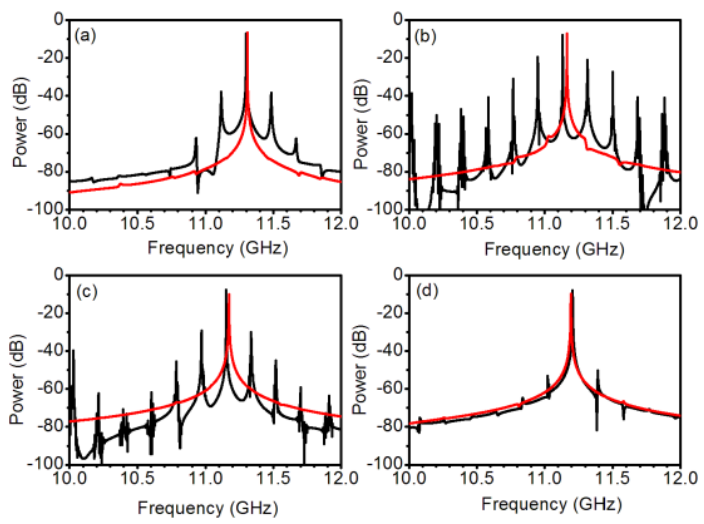

Fig. 9 Numerical simulation of RF spectra of the VCSEL with single feedback (black curve) and double feedback (red curve). Feedback phase $\phi$ from the short cavity (a) 0 ; (b) $0.2 \pi$; (c) $0.4 \pi$; (d) $\pi$.

\section{CONCLUSION}

We have studied the effect of optical feedback on the linewidth and stability of photonic microwave generated based on P1 
dynamics in an optically injected single-mode VCSEL subject to optical feedback. The results show that both single feedback and double feedback can reduce the linewidth. However, the feedback phase in the single feedback configuration must be carefully adjusted to suppress the side peaks. The linewidth in the double feedback configuration can, with optimal feedback phase, be reduced to less than one tenth of linewidth without the optical feedback. Numerical simulation confirm that the side peak suppression is sensitive to the feedback phase for the single feedback and are in good agreement with the results obtained in the experiment. For the parameters we used, anti-phase feedback favors the side peaks suppression, and $0.2 \pi$ feedback phase triggers many side peaks with high amplitude. We also investigate the stability of photonic microwave, which is quantified by measuring the microwave frequency range using a long sweep time of the radio frequency spectrum analyzer. The results indicate that optical feedback can also improve the stability of the generated microwave.

\section{ACKNOWLEDGMENT}

C. P. Xue thanks the financial support from China Scholarship Council (201706070007). We thank Prof. Guanqiong Xia from school of physical science and technology, Southwest University, Chongqing 400715, for her very useful advice.

\section{REFERENCES}

[1] N. Dagli, "Wide-bandwidth lasers and modulators for RF photonics," IEEE Trans. Microw. Theory Tech. vol. 47, no. 7, pp. 1151-1171, Jul. 1999.

[2] A. J. Seeds and K. J. Williams, "Microwave photonics," J. Lightw Technol. vol. 24, no., 12, pp. 4628-4641, Dec. 2006

[3] J. Capmany and D. Novak, "Microwave photonics combines two worlds," Nat. Photonics, vol. 1, pp. 319-330, Jun. 2007.

[4] J. P. Yao, "Microwave photonics," J. Lightw. Technol., vol. 27, no. 3, pp. 314-335, Mar. 2009, DOI. 10.1109/JLT.2008.2009551

[5] T. L. Wang, H. W. Chen, M. H. Chen, J. Zhang, S. H. Xie, "High-Spectral-Purity Millimeter-Wave Signal Optical Generation," $J$. Lightw Technol. vol. 27, no., 12, pp. 2044-2051, Jun. 2009

[6] X. K. Liu, W. Pan, X. H. Zou, D. Zheng, L. S. Yan, B. Luo, and B. Lu, "Photonic Generation of Triangular-Shaped Microwave Pulses Using SBS-Based Optical Carrier Processing ", J. Lightw. Technol., vol. 32, no. 20, pp. 3797-3802, Oct. 2014, DOI. 10.1109/JLT.2014.2313349

[7] D. Novak, R. B. Waterhouse, A. Nirmalathas, C. Lim, P. A. Gamage, T. R. Clark, Jr., M. L. Dennis, and J. A. Nanzer, "Radio-Over-Fiber Technologies for Emerging Wireless Systems," IEEE J. Quantum Electron., vol. 52, no. 1, Art. No. 0600311, Jan. 2016.

[8] X.-Q. Qi and J.-M. Liu, "Photonic Microwave Applications of the Dynamics of Semiconductor Lasers," IEEE J. Sel. Top. Quantum Electron. vol. 17, no. 5, pp. 1198-1211, 2011.

[9] S.-C. Chan, S.-K. Hwang, and J.-M. Liu, "Period-one oscillation for photonic microwave transmission using an optically injected semiconductor laser," Opt. Express, vol. 15, no. 22, pp. 14921-14935, Oct. 2007

[10] M. Pochet, T. Locke, and N. G. Usechak, "Generation and Modulation of a Millimeter-Wave Subcarrier on an Optical Frequency Generated via Optical Injection," IEEE Photonics J. vo. 4, no. 5, pp. 1881-1891, Oct. 2012.

[11] T. B. Simpson, J. M. Liu, M. Almulla, N. G. Usechak, and V. Kovanis, "Linewidth sharpening via polarization-rotated feedback in optically injected semiconductor laser oscillators," IEEE J. Sel. Top. Quantum Electron. vol. 19, no. 4, Art. no. 1500807, Jul./Aug. 2013.

[12] Y.-H. Hung and S. K. Hwang, "Photonic microwave amplification for radio-over-fiber links using period-one nonlinear dynamics of semiconductor lasers s," Opt. Lett. vol. 38, no. 17, pp. 3355-3358, 2013.
[13] A. Hurtado, I. D. Henning, M. J. Adams, and L. F. Lester, "Generation of tunable millimeter-wave and $\mathrm{THz}$ signals with an optically injected quantum dot distributed feedback laser," IEEE Photonics J. vol. 5, no. 4, Art. no. 5900107, 2013.

[14] C. Wang, R. Raghunathan, K. Schires, S.-C. Chan, L. F. Lester, and F. Grillot, "Optically injected InAs/GaAs quantum dot laser for tunable photonic microwave generation," Opt. Lett. vol. 41, no. 6, pp. 1153-1156, 2016.

[15] R. Michalzik (eds), VCSELs - Fundamentals, Technology and Applications of Vertical-Cavity Surface-Emitting Lasers, Springer Series in Optical Sciences, vol. 166, 2013, ch.2.

[16] A. Quirce, A. Valle, H. Lin, D. W. Pierce, and Y. Zhang, "Photonic generation of high-frequency microwave signals utilizing a multi-transverse-mode vertical-cavity surface-emitting laser subject to two-frequency orthogonal optical injection," J. Opt. Soc. Am. B, vol. 29, no.12, pp. 3259-3270, 2012.

[17] P. Perez, A. Quirce, A. Valle, A. Consoli, I. Noriega, L. Pesquera, and I. Esquivias, "Photonic generation of microwave signals using a single-mode VCSEL subject to dual-beam orthogonal optical injection," IEEE Photonics J. vol. 7, no. 1, Art. no. 5500614, 2015.

[18] B. Sun, J. -G. Wu, S. -T. Wang, Z. -M. Wu and G. -Q. Xia, "Theoretical and experimental investigation on the narrow-linewidth photonic microwave generation based on parallel polarized optically injected $1550 \mathrm{~nm}$ vertical-cavity surface-emitting laser," Acta Phys. Sin. vol. 65 , no. 1 , Art. no. 014207,2016

[19] S. Ji, Y. Hong, P. S. Spencer, J. Benedikt, and I. Davies, "Broad tunable photonic microwave generation based on period-one dynamics of optical injection vertical-cavity surface-emitting lasers," Opt. Express, vol. 25, no. 17, pp. 19863-19871, Aug. 2017.

[20] H. Lin, A. Ourari, T. Huang, A. Jha, A. Briggs and N. Bigagli, "Photonic microwave generation in multimode VCSELs subject to orthogonal optical injection," J. Opt. Soc. Am. B., vol. 34, no. 11, pp. 2381-2389, Nov. 2017

[21] J.-P. Zhuang and S.-C. Chan, "Phase noise characteristics of microwave signals generated by semiconductor laser dynamics," Opt. Express, vol. 23, no. 3, pp. 2777-2797, Feb. 2015.

[22] J.-P. Zhuang and S.-C. Chan, "Tunable photonic microwave generation using optically injected semiconductor laser dynamics with optical feedback stabilization," Opt. Lett. vol. 38, no. 3, pp. 344-346, Feb. 2013.

[23] C.-H. Cheng, C.-W. Lee, T.-W. Lin, and F.-Y. Lin, "Dual-frequency laser Doppler velocimeter for speckle noise reduction and coherence enhancement," Opt. Express, vol. 20, no. 18, pp. 20255-20265, Aug. 2012.

[24] T. B. Simpson and F. Doft, "Double-locked laser diode for microwave photonics applications," IEEE Photonics Technol. Lett. vol. 11, no.11, pp.1476-1478, Nov. 1999.

[25] L. Fan, G. Xia, J. Chen, X. Tang, Q. Liang, and Z. Wu, "High-purity $60 \mathrm{GHz}$ band millimeter-wave generation based on optically injected semiconductor laser under subharmonic microwave modulation," Opt. Express, vol. 24, no. 16, pp. 18252-18265, 2016.

[26] Yu-Shan Juan and Fan-Yi Lin, "Photonic Generation of Broadly Tunable Microwave Signals Utilizing a Dual-Beam Optically Injected Semiconductor Laser," IEEE Photonics J. vol. 3, no. 4, pp.644-650, Aug. 2011.

[27] A. Quirce and A. Valle, "High-frequency microwave signal generation using multi-transverse mode VCSELs subject to two-frequency optical injection.," Opt. Express, vol. 20, no. 12, pp. 13390-13401, Mar. 2012.

[28] S.-C. Chan, and J.-M. Liu, "Tunable narrow-linewidth photonic microwave generation using semiconductor laser dynamic," IEEE J. Sel. Top. Quantum Electron. vol. 10, no.5, pp. 1025-1032, Sept./Oct. 2004

[29] K.-H. Lo, S.-K. Hwang, and S. Donati, "Optical feedback stabilization of photonic microwave generation using period-one nonlinear dynamics of semiconductor lasers," Opt. Express, vol. 22, no. 15, pp.18648-18661, Jul. 2014.

[30] A. Murakami, K. Kawashima, and K. Atsuki, "Cavity resonance shift and bandwidth enhancement in semiconductor lasers with strong light injection," IEEE J. Quantum Electron., vol. 39, no. 10, pp. 1196-1204, Oct. 2003 